\title{
Work-in-Progress-Enhancing Training in Virtual Reality with Hand Tracking and a Real Tool
}

\section{Conference Paper}

\section{Author(s):}

Gisler, Joy; Holzwarth, Valentin; Hirt, Christian (D); Kunz, Andreas (DD

Publication date:

2021-06

Permanent link:

https://doi.org/10.3929/ethz-b-000493694

Rights / license:

In Copyright - Non-Commercial Use Permitted

Originally published in:

https://doi.org/10.23919/ilrn52045.2021.9459332 


\section{Work-in-Progress-Enhancing Training in Virtual Reality with Hand Tracking and a Real Tool}

\author{
$1^{\text {st }}$ Joy Gisler \\ Innovation Center Virtual Reality \\ ETH Zurich \\ Zurich, Switzerland \\ gj@ethz.ch \\ $3^{\text {rd }}$ Christian Hirt \\ Innovation Center Virtual Reality \\ ETH Zurich \\ Zurich, Switzerland \\ hirtc@ethz.ch
}

\author{
$2^{\text {nd }}$ Valentin Holzwarth \\ Institute of Information Systems \\ University of Liechtenstein \\ Vaduz, Liechtenstein \\ valentin.holzwarth@uni.li \\ $4^{\text {th }}$ Andreas Kunz \\ Innovation Center Virtual Reality \\ ETH Zurich \\ Zurich, Switzerland \\ kunz@iwf.mavt.ethz.ch
}

\begin{abstract}
The main goal of Virtual Training Environments (VTEs) is to maximize training effectiveness, which can be achieved by increasing the degree of immersion. While prior work mainly focused on the visual, auditory, and navigational aspects of immersion, proprioceptive aspects may be particularly important. In this work-in-progress paper, we explore this potential by implementing an industrial VTE, which can be interacted with, using VR gloves and a tracked real tool. Further, we evaluate the VTE in a pilot study with industry apprentices, providing initial evidence that participants experienced high presence and low task load, while being generally satisfied with the training.

Index Terms-virtual reality, visualization, tracked objects, training simulator, haptics
\end{abstract}

\section{INTRODUCTION}

The unique ability to provide highly immersive experiences makes Virtual Reality (VR) a promising technology for corporate education and training procedures. Training industrial tasks virtually comes with a variety of benefits, such as avoiding the waste of real raw material, reduced risk of injury, as well as location and language independence. In this context, the degree of immersion plays a key role in achieving high training effectiveness and usability [1], [2]. The most straightforward way to reach higher degrees of immersion is to increase the visual fidelity (e.g. by using Head-Mounted Displays (HMDs) with high resolution [3]). However, in the context of industrial VR training, haptic feedback may play a more dominant role, as most tasks to be trained heavily rely on workers' dexterity. Thus, the inclusion of real objects and tools into Virtual Training Environments (VTEs) might not only be appealing, but essential to achieve high training effectiveness.

Generally, industrial tasks require workers to possess procedural knowledge [4] (e.g. executing the required steps in the correct sequence) and the corresponding psychomotor skills [5] (e.g. muscle memory and dexterity). While procedural knowledge can be trained in VTEs with low immersion (e.g. controller-based, no real-walking), the acquisition of psychomotor skills requires a "learning-by-doing" approach, including preferably real, manual interaction with tracked real objects or tools. However, prior works have either focused on other VTE contexts (e.g. sports training [6], [7]) or other immersive features (e.g. display fidelity [3]).

In this work, we develop and preliminarily evaluate a VTE for an industrial task, including manual interaction using VR gloves and a tracked real tool. This allows trainees not only to acquire procedural knowledge, but also psychomotor skills including the correct positioning and operation of the equipment required to accomplish the task.

We organize the remainder of this paper as follows: In section II. we outline the related work on real objects and manual interaction in the context of industrial VTEs and beyond. Subsequently, in section III we describe the methodology consisting of the task to be trained, the VTE itself, the employed apparatus and the integration of the real tracked tool. In section IV, we present and discuss the results of our pilot study, whereas we conclude the paper and address potential directions for future research in section V

\section{STATE OF THE ART}

VTEs have been studied in various industrial contexts, such as assembly [1], safety [3], [8], and maintenance [9]. Prior works discussed multiple VTEs and compared fully immersive approaches to other training media (e.g. smartphones or printed materials [8]) and investigated on training transfer to corresponding real tasks (e.g. tank maintenance procedures [9] or sequential assembly [2]). Further, the influence of certain immersive features on training effectiveness has been studied (e.g. vision [3], or interaction and navigation [2]). Beyond the industrial context, the integration of real objects in VTEs has been investigated, e.g. a real tracked sphere for process model exploration [10], a real baseball in a sports training simulator [6], or a real putter in a golf VTE [7]. While users' interest was significantly higher for the real tracked sphere in a process model exploration VTE, it did not increase training 


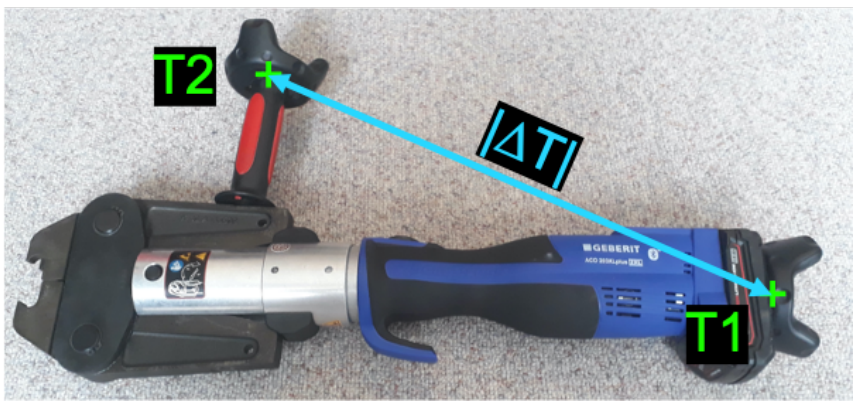

(a) Pipe pressing tool with adapter and trackers

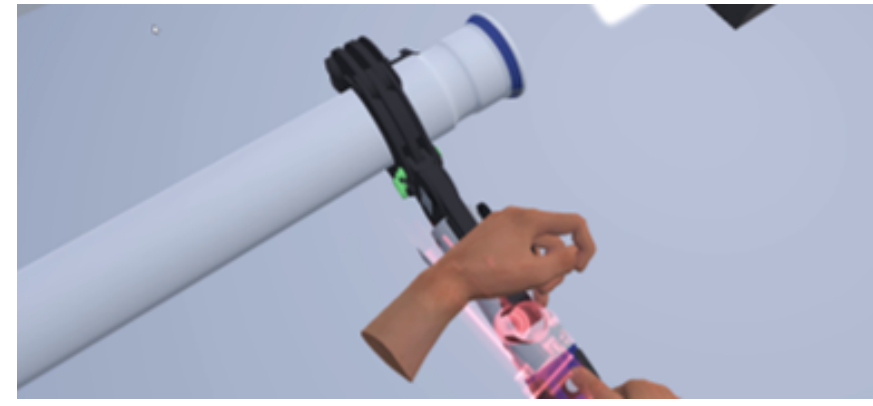

(b) Red hologram appears if the tool is positioned incorrectly

Fig. 1: Tracked tool (left) and an instructional feature (right).

effectiveness compared to a conventional controller setup [10]. However, in the context of the golf VTE, a real tracked putter resulted in significantly better user performance compared to the controller setup [7]. Drawing from these promising prior implementations of real objects in non-industrial VTEs, we hypothesize that these benefits could also be harnessed in our context, which is industrial training.

\section{Methodology}

To improve the external validity, research on training in VR should cover more industry-related tasks and should be evaluated within authentic settings, i.e. the actual target group of the VTE [1]. Therefore, we collaborated with an industry partner from the field of building services engineering to identify a suitable task (see subsection III-A). Next, we implemented a VTE to train this task (see subsection III-B) and integrated the real tool (see subsection III-D). Finally, we conducted a pilot study within an authentic setting, which consisted of industry apprentices (see section subsection III-E).

\section{A. Pipe Pressing Task}

The task at hand is the pressing of steel pipes with a diameter of $108 \mathrm{~mm}$ using a handheld pressing tool, which is shown in Fig. 1a The pressing procedure consists of four specific steps that need to be conducted in a predefined sequence (i.e. procedural knowledge), as well as the correct positioning and operation of the tool (i.e. psychomotor skills). Training this task is relevant for plumbers and heating engineers, since improper execution could damage the equipment, which would eventually lead to costly disruptions on job sites.

However, this task is currently not trained in vocational schools due to high costs for raw material, amounting to hundreds of USD per training session. Consequently, the pipe pressing task is particularly appealing for VR training, requiring both procedural knowledge and psychomotor skills, while allowing for substantial cost savings.

\section{B. Virtual Training Environment}

The VTE was implemented in Unity (Version 2019.4.16f1), including models of the required equipment, text-based instructions that guide a trainee through the process, and generic virtual objects (e.g. walls, table, and lights). Furthermore, an audiovisual instructional feature notified trainees, whenever they were handling the tool improperly, i.e. when the tool was not positioned correctly, the apprentices would hear a beeping sound and see a flashing red hologram of the tool's correct placement (see Fig. 1b). A side-by-side comparison of the VTE rendered in the HMD and a trainee experiencing it is shown in Fig. 2.

\section{Apparatus}

The VTE was rendered at $90 \mathrm{~Hz}$ on a computer with an Intel i7-9700K, an Nvidia RTX 2080, and 32 gigabytes RAM. We used an HTC VIVE Pro Eye HMD and two SteamVR base stations 2.0 to allow real walking in a $6 \mathrm{~m} \times 3 \mathrm{~m}$ roomscale tracking space. The hands and fingers of the trainees were tracked with a pair of sensoryx VRfree Gloves [11]. The VRfree Gloves were integrated using the VRfree Unity SDK provided by their vendor. The pressing tool was tracked using two HTC VIVE Trackers 2018. Both trackers were attached to the tool using $1 / 4$ " photographic screws.

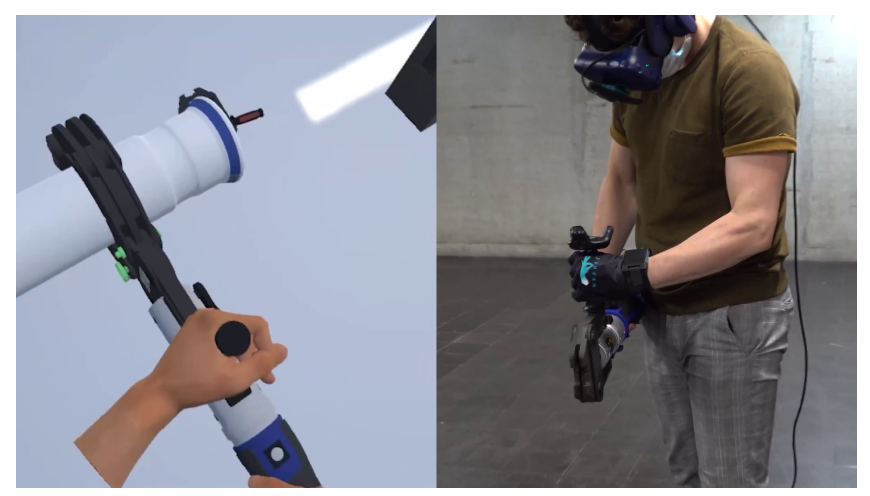

Fig. 2: Side-by-side comparison of the VTE rendered in the HMD (left) and a trainee experiencing it (right).

\section{Real Tool Integration}

To integrate the handheld pressing tool in the VTE, two core functionalities are required: i) tracking the tool's position with respect to the user and ii) tracking the manipulation of the pressing tool's adapter (i.e. opening and closing, see Fig. 31. This was accomplished using two HTC VIVE Trackers 2018 
(see Fig. 1a). The first tracker (T1) was mounted to the back of the pressing tool to determine the position of the pressing tool with adapter in the VTE. The second tracker (T2) does not contribute to the tracking of the pressing tool's position in the VTE. It was used exclusively to map the manipulation of the pressing tool adapter (i.e. opening and closing) into the VTE. Based on the distance between $\mathrm{t}$ he two trackers $|\Delta T|$, the claws of the pressing tool's adapter in the VTE were either closed (see Fig. 3a) or open (see Fig. 3b). To match the appearance of its real counterpart, the adapter in the VTE fully opened if $|\Delta T|$ was smaller than an empirically determined threshold and remained closed otherwise.

\section{E. Pilot Study}

The pilot study included 17 participants and was conducted at a vocational school. All participants were male sanitary apprentices. Their mean age was 20.35 years with a standard deviation (SD) of 5.51 years. The study was conducted in single-user sessions, which lasted 45 minutes each on average. After being informed about the study procedure, participants signed a consent form and filled out a demographic questionnaire. Afterwards, the provided hardware (HTC VIVE Pro Eye, VRFree Gloves [11], and tracked tool) was introduced and the tutorial scene was started, which allowed participants to familiarize themselves with the virtual environment. Then, the participants proceeded to the actual VTE. Finally, participants answered standardized questionnaires concerning their sense of presence (SUS presence, 6 items), task load (NASA TLX, 6 items), and learner satisfaction (LS, 4 items) after completing the VTE.

\section{Preliminary Results and Discussion}

On average, participants scored their sense of presence in the VE at $4.65(\mathrm{SD}=1.06)$ on a Likert scale from 1 to 7 (higher is better). Participants felt an average task load of 18.88 (SD = 7.82) out of 100. Finally, participants reported an average satisfaction with the VTE of $5.79(\mathrm{SD}=0.73)$ out of 7 .

Our application invoked a reasonably high sense of presence. Our results indicate that - despite the tool's considerable weight of approximately $10 \mathrm{~kg}$ - the integration of the tracked tool did not put too much strain on participants' perceived task load. The reported learner satisfaction shows that enhancing a VTE with a real tracked tool and hand tracking is generally appreciated by trainees. This is also supported by informal feedback from participants that we received after the study sessions.

\section{CONCLUSION AND Future WORK}

In this paper, we developed and preliminarily evaluated a VTE for an industrial pipe pressing task, including VR gloves and a tracked real tool. By these means, trainees can acquire both, procedural knowledge and psychomotor skills, which are needed to successfully accomplish the pressing procedure. Further, we conducted a pilot study with $\mathrm{N}=17$ participants, which provided initial evidence that the VTE satisfies the

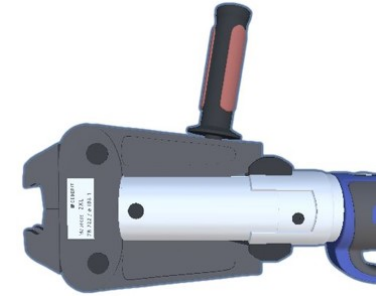

(a) Tool adapter closed

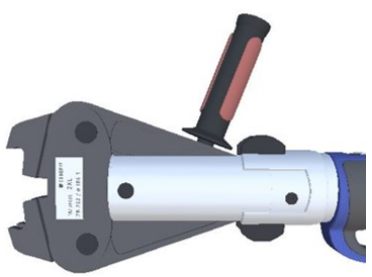

(b) Tool adapter opened
Fig. 3: The model of the pressing tool in different states, showing only the front end of the tool and the adapter.

trainees, while evoking a reasonable sense of presence and imposing not too much task load on them.

Future work should focus on measuring training effectiveness and validating the implemented VTE, e.g. by comparing it to an implementation using commercially available VR controllers only. Finally, also the potential of providing instructions in a language-independent way to make it more accessible for trainees with language deficiencies should be investigated.

\section{ACKNOWLEDGMENT}

This work was partly funded by the Innosuisse 43670.1 INNO-ICT project. Further, the authors wish to express their gratitude towards Müller Wüst AG for project initiation and partnership, Geberit AG for equipment provision and support, as well as Berufsschule Lenzburg for hosting the pilot study.

\section{REFERENCES}

[1] C. Hirt, V. Holzwarth, J. Gisler, J. Schneider, and A. Kunz, "Virtual learning environment for an industrial assembly task," in 9th Int. Conf. Consum. Electron. (ICCE 2019), pp. 337-342.

[2] J. Gisler, C. Hirt, A. Kunz, and V. Holzwarth, "Designing virtual training environments: Does immersion increase task performance?" in 2020 Int. Conf. Cyberworlds (CW), pp. 125-128.

[3] F. Buttussi and L. Chittaro, "Effects of Different Types of Virtual Reality Display on Presence and Learning in a Safety Training Scenario," IEEE Trans. Vis. Comput. Graphics, vol. 24, no. 2, pp. 1063-1076, 2018.

[4] G. Makransky, T. S. Terkildsen, and R. E. Mayer, "Adding immersive virtual reality to a science lab simulation causes more presence but less learning," Learn. Instruct., vol. 60, pp. 225-236, 2019.

[5] H. Kaufman, R. Wiegand, and R. Tunick, "Teaching surgeons to operate-principles of psychomotor skills training," Acta Neurochirurgica, vol. 87 , no. 1 , pp. 1-7, 1987.

[6] S. Lontschar, D. Deegan, I. Humer, K. Pietroszek, and C. Eckhardt, "Analysis of Haptic Feedback and its Influences in Virtual Reality Learning Environments," in Proc. 6th Int. Conf. Immersive Learn. Res. Netw., iLRN 2020, pp. 171-177.

[7] A. Franzluebbers and K. Johnsen, "Performance Benefits of HighFidelity Passive Haptic Feedback in Virtual Reality Training," in Proc. Symp. Spatial User Interact. (SUI '18), 2018, pp. 16-24.

[8] F. Buttussi and L. Chittaro, "A Comparison of Procedural Safety Training in Three Conditions: Virtual Reality Headset, Smartphone, and Printed Materials," IEEE Trans. Learn. Technol., pp. 1-15, 2020.

[9] F. Ganier, C. Hoareau, and J. Tisseau, "Evaluation of procedural learning transfer from a virtual environment to a real situation: a case study on tank maintenance training," Ergonom., vol. 57, no. 6, pp. 828-843, 2014.

[10] A. Zenner, A. Makhsadov, S. Klingner, D. Liebemann, and A. Kruger, "Immersive process model exploration in virtual reality," IEEE Trans. Vis. Comput. Graphics, vol. 26, no. 5, pp. 2104-2114, 2020.

[11] Sensoryx AG. "vrfree - technical details." accessed mar. 20, 2021. [Online]. Available: https://www.sensoryx.com/company/technology/ 